\title{
Extrapyramidal involvement in amyotrophic lateral sclerosis: backward falls and retropulsion
}

\author{
Joy Desai, Michael Swash
}

\begin{abstract}
Three patients with sporadic amyotrophic lateral sclerosis (ALS) presented with a history of backward falls. Impaired postural reflexes and retropulsion accompanied clinical features of ALS. Hypokinesia, decreased arm swing, and a positive glabellar tap were noted in two of these three patients. Cognitive impairment, tremor, axial rigidity, sphincter dysfunction, nuchal dystonia, dysautonomia, and oculomotor dysfunction were absent. Brain MRI disclosed bilateral $\mathrm{T} 2$ weighted hyperintensities in the internal capsule and globus pallidus in one patient. Necropsy studies performed late in the course of ALS have shown degeneration in extrapyramidal sites-for example, the globus pallidus, thalamus, and substantia nigra. Clinically, backward falls and retropulsion may occur early in ALS. This may reflect extrapyramidal involvement.
\end{abstract}

(F Neurol Neurosurg Psychiatry 1999;67:214-216)

Keywords: amyotrophic lateral sclerosis; backward falls; retropulsion; globus pallidus

We found backward falls and retropulsion due to postural instability early in the course of the illness in three of a consecutive series of 22 patients with sporadic amyotrophic lateral sclerosis (ALS). We report the clinical, laboratory, and neuro-imaging features and discuss the possible pathogenic basis of these extrapyramidal features.

The Department of Neurology, The Royal London Hospital, London E1 1BB, UK J Desai

M Swash

Correspondence to: Professor Michael Swash, Department of Neurology, The Royal London Hospital, Whitechapel, London E1 1BB, UK. Telephone 0044 171377 7472; fax 0044171 377 7318; email

mswash@mds.qmw.ac.uk and mswash@btinternet.com

Received 9 November 1998 and in revised form 20 January 1999

Accepted 24 January 1999

\section{Case reports}

PATIENT 1

A 60 year old man, previously well, developed slurred speech. He complained of stiffness in both legs and frequent leg cramps. A month later while opening a door he fell backwards and incurred a minor scalp injury. Subsequently, he fell backwards twice while arising from a chair. He noticed frequent twitching of muscles in his arms. Six months later, he developed difficulty in swallowing food and sometimes choked on drinks. He took an increasingly longer time to complete daily activities such as dressing and bathing. There was no history of cognitive decline or tremor. Sensory, sphincter, visual, and autonomic functions were normal. There was no family history of neurological illness.

General examination and vital indices were normal. There was no postural hypotension. There was a spastic dysarthria, and the palate moved poorly. The jaw jerk was brisk and the tongue was wasted. Fasciculations were seen in the tongue and limb muscles. There was asymmetric wasting in the distal muscles of both upper limbs. Power was 3/5 (MRC) distally, and $4 / 5$ proximally in the upper limbs. In the legs, power was $4 / 5$ in all muscles. Spasticity was found in all four limbs, with mild cogwheel rigidity at the wrists. There was hypokinesia of the face and posture. The speed of rapid rhythmic finger and toe tapping was reduced but there was no rest tremor. Postural reflexes were impaired. When gently pulled backwards, even after a prior warning, he could not maintain stability and toppled backwards. There was no adaptation to repetition of this manoeuvre. A gentle push on the chest resulted in a backward fall. He could maintain an upright stance and did not fall on being pushed forwards or laterally. All deep tendon reflexes were exaggerated and plantar responses were bilaterally extensor. Hoffman's sign was positive in both upper limbs. The rest of the neurological assessment was normal. The haematology, biochemistry, erythrocyte sedimentation rate, immunoelectrophoresis, thyroid functions, CSF, and MRI of the brain were normal. Nerve conduction studies were normal. Concentric needle EMG disclosed fasciculations, positive sharp waves, and evidence of chronic partial denervation with reinnervation in proximal and distal muscles of all four limbs.

One year later, upper limb strength had decreased to $3 / 5$ (MRC). Power in the lower limbs had not changed although fasciculations were more obvious. Swallowing and speech had deteriorated. Gentle forward and lateral pushes now resulted in uncontrolled falls, resistant to adaptation. There was more marked hypokinesia and glabellar tap was positive. Cognition remained normal. The postural reflexes did not improve after a 6 week trial of levodopa in a dose of $1 \mathrm{~g} /$ day.

PATIENT 2

A 65 year old woman presented with a 3 month history of stiffness and weakness of the right arm. She had difficulty in using the right hand 
to open bottles, turn keys in locks, and to button clothes. She fatigued excessively. Five months later she fell backwards while lifting a pot in her garden and on three further occasions she fell backwards while working in her kitchen. She noted progressive weakness, and wasting in the right hand with twitching of muscles in both arms and occasionally in the legs. Her speech slurred when she spoke quickly. Her medical history was unremarkable. Her mother had died aged 87 years with Alzheimer's dementia.

General examination was normal. Glabellar tap was positive. Her speech was slurred. The jaw jerk was brisk and palatal movements were normal. Lip smacking and tongue movements in all directions were slow. Fasciculations were noted in both arms and the left leg but not in the tongue. There was wasting in the left deltoid, triceps, and distal muscles of the right hand and forearm. There was spasticity in all four limbs with some cogwheel rigidity at the wrists. Power was MRC 4/5 in the left upper limb and both lower limbs. In the proximal muscles of the right arm power was MRC 3/5 and $2 / 5$ to $1 / 5$ in the distal muscles of the right hand. All the deep tendon reflexes were exaggerated and both plantar responses were extensor. Hoffman's sign was positive on the left side. Postural reflexes were impaired. A gentle backward pull, after prior warning, resulted in marked instability and an uncontrolled backward fall. This did not adapt with repetition. Similar forward or lateral pushes did not result in instability or falls. The gait was spastic, and arm swing was absent on the right. There was no cognitive impairment or autonomic dysfunction.

Haematology, biochemistry, thyroid function tests, erythrocyte sedimentation rate, serum immunoelectrophoresis, and CSF examination were normal. Motor and sensory nerve conduction was normal. Concentric

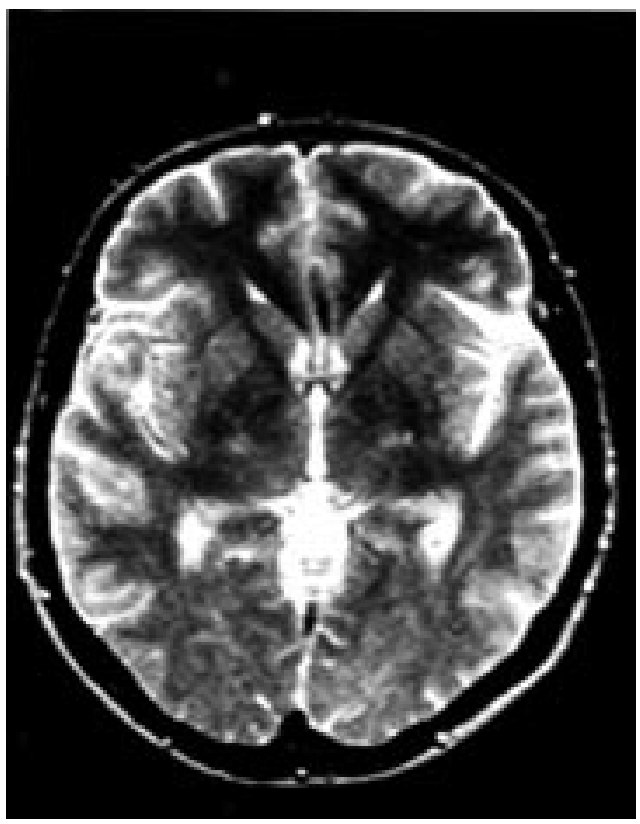

Axial T2 weighted MRI: hyperintense signal in the internal capsule extending into the globus pallidus. needle EMG disclosed profuse fasciculations in both upper limbs and sporadic fasciculation in the proximal muscles of the lower limbs. There was chronic partial denervation in both upper limbs and in proximal muscles of the lower limbs. Brain MRI showed bilateral T2 weighted hyperintensities in the internal capsule extending into the globus pallidus (figure).

\section{PATIENT 3}

A 56 year old woman presented with a 5 month history of proximal weakness in the right arm. She noticed twitching of muscles in both arms. Her legs felt stiff and she walked more slowly. She had a backward fall while opening her car door and on three other occasions she fell backwards in her garden while bending to trim a hedge.

The general examination and vital indices were normal. There was spasticity in all four limbs. The deltoid and dorsal interossei muscles were wasted. Fasciculations were visible in both arms and proximal muscles of the legs. Power was MRC 3/5 in the deltoids, biceps, and brachioradialis bilaterally. In other muscles power was $4 / 5$. In the distal muscles of the lower limbs power was 5/5. The deep tendon reflexes were brisk and both plantar responses were extensor. Postural reflexes were impaired and retropulsion was easily elicited. There was no adaptation to repetition. There was no propulsion or lateropulsion. Cognition and coordination were normal.

Haematology, biochemistry, erythrocyte sedimentation rate, CSF, serum immunoelectrophoresis, thyroid functions, and MRI of the brain were normal. Nerve conduction studies were normal. Concentric needle EMG showed fasciculations and positive sharp waves in the upper limbs. There was chronic partial denervation with reinnervation in proximal and distal muscles of the upper limbs, and proximal muscles of the lower limbs.

\section{Discussion}

Our patients fulfilled the Airlie House criteria (1998) for the diagnosis of ALS (<http:// www.wfnals.org/Airlie criteria/>; to be published). However, in our three patients backward falls, a feature of extrapyramidal dysfunction, occurred early in the illness. Their postural reflexes were impaired and retropulsion was a striking clinical feature. Brain MRI in patient 2 showed T2 weighted hyperintensities in the internal capsule extending into the globus pallidus (figure). This probably reflects gliosis secondary to neuronal degeneration in these nuclei. Similar MRI features have been reported in Japanese patients with ALS. ${ }^{1}$

Striking impairment of postural reflexes resulting in backward falls occurs in progressive supranuclear palsy and multiple system atrophy. The absence of frontal lobe dysfunction, dysautonomia, nuchal dystonia, and cerebellar and oculomotor dysfunction ruled out these diagnostic possibilities in our patients.

The pathophysiology of impairment of postural reflexes in disorders of the basal ganglia is complex. ${ }^{2}$ Martin suggested that in 
“encephalitis lethargica" impairment of postural reflexes is attributable to degeneration in the globus pallidus. ${ }^{34} \mathrm{~A}$ patient with carbon monoxide poisoning who had bilateral selective damage to the globus pallidus, fell slowly backwards on being gently tapped on the shoulder. ${ }^{5}$

Asymptomatic, subclinical degeneration in the midbrain dopaminergic neurons has been shown by PET, ${ }^{67}$ and SPECT, ${ }^{8}$ in both sporadic and familial ALS. Degeneration in the midbrain dopaminergic neurons has also been detected in a transgenic mouse model of familial ALS. ${ }^{9}$ Neurofibrillary tangles have been described in the globus pallidus, substantia nigra, and locus coeruleus of a patient with ALS who had a point mutation at codon 113 in exon 4 of the SOD 1 gene. ${ }^{10}$ Sporadic ALS with widespread neuronal degeneration in the basal ganglia, including the globus pallidus, has been reported in Japan where survival is often prolonged by assisted ventilation. ${ }^{11}{ }^{12}$ Necropsy studies are inevitably done late in the course of the illness. In our patients with ALS, postural instability, a feature of extrapyramidal involvement, developed early in the illness.

Eisen and Calne suggested that parkinsonism, with or without dementia was associated with ALS at the same time in the course of the disease in $5 \%$ of cases. ${ }^{13}$ In chromosome 17 associated frontotemporal dementia and Guamanian ALS, dementia of frontal lobe type, parkinsonism, and ALS occur in varying combinations. However, dementia was not seen in our patients. Published reports of parkinsonism associated with sporadic ALS have emphasised the presence of rigidity, bradykinesia, tremor, and gait disorder. ${ }^{14}{ }^{15}$ We report the early impairment of postural reflexes resulting in backward falls and retropulsion in three patients with sporadic ALS. The presence of these extrapyramidal features should not invalidate a clinical diagnosis of ALS.

1 Kato S, Hayashi H, Yagishita A. Involvement of the frontotemporal lobe and limbic system in amyotrophic lateral clerosis: as assessed by serial computed tomography and magnetic resonance imaging. F Neurol Sci 1993; 116:52-8.

2 Bloem BR. Postural instability in Parkinson's disease. Clin Neurol Neurosurg 1992;94(suppl):S41-5.

3 Martin JP. The basal ganglia and posture. London: Pittman, 1967.

4 Martin JP, Hurwitz LJ, Finlayson MH. The negative symptoms of basal ganglia disease. Lancet 1962;ii:1, 62 .

5 Haaxma $\mathrm{R}$, van Boxtel A, Brouwer WH, et al. Motor function in a patient with bilateral lesions of the globus pallidus. Mov Disord 1995;10:761-77.

6 Takahashi H, Snow BJ, Bhatt MH, et al. Evidence of a dopaminergic deficit in sporadic amyotrophic lateral sclerosis on positron emission scanning. Lancet 1993;324: sclerosis on

7 Przedborski S, Dhawan V, Donaldson DM, et al. Nigrostriatal dopaminergic function in familial amyotrophic lateral sclerosis patients with and without copper/zinc superoxide sclerosis patients with and without copper/zinc sup
dismutase mutations. Neurology 1996;47:1546-51.

8 Borasio GD, Linke R, Schwarz J, et al. Dopaminergic deficit in amyotrophic lateral sclerosis assessed with [I-123] IPT single photon emission computed tomography. $f$ Neurol Neurosurg Psychiatry 1998;65:263-5.

9 Kostic V, Gurney ME, Deng HX, et al. Midbrain dopaminergic neuronal degeneration in a transgenic mouse model of familial amyotrophic lateral sclerosis. Ann Neurol 1997;41:497-504.

10 Orrell RW, King AW, Hilton DA, et al. Familial amyotrophic lateral sclerosis with a point mutation of SOD-1: intrafamilial heterogeneity of disease duration associated with neurofibrillary tangles. $\mathcal{F}$ Neurol Neurosurg Psychiatry 1995;59:266-70.

11 Iwanaga K, Hayashi S, Okaye M, et al. Neuropathology of sporadic amyotrophic lateral sclerosis of long duration. $\mathcal{f}$ Neurol Sci 1997;146:139-43.

12 Sasaki S, Tsutsumi Y, Yamane $\mathrm{K}$, et al. Sporadic amyotrophic lateral sclerosis with extensive neurological amyotrophic lateral sclerosis with extensive neurol

13 Eisen A, Calne D. Amyotrophic lateral sclerosis, Parkinson's disease and Alzheimer's disease: phylogenetic disorders of the human neocortex sharing many characteristics. Can $\mathcal{F}$ Neurol Sci 1992;19:117-23.

4 Qureshi AI, Wilmot G, Dihenia B, et al. Motor neuron disease with parkinsonism. Arch Neurol 1996;53:987-91.

15 Williams TL, Shaw PJ, Lowe J, et al. Parkinsonism in motor neuron disease: case report and literature review. Acta Neuropathologica 1995;89:275-83. 